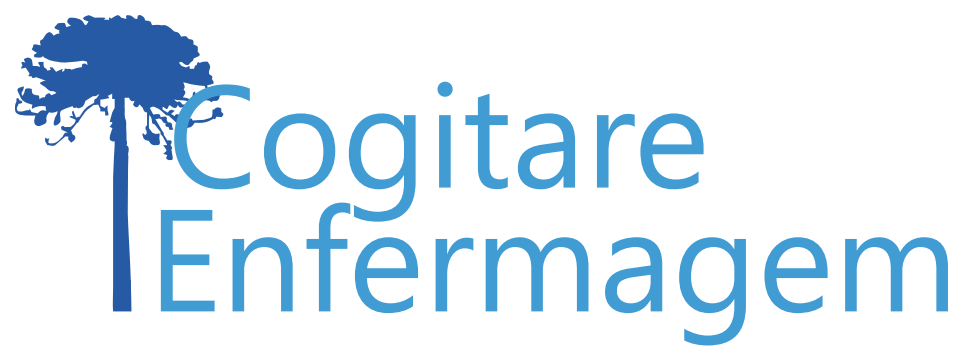

\title{
APRENDIZAGEM EXPERIENCIAL E DIRETRIZES CURRICULARES NACIONAIS DE ENFERMAGEM: REVISÃO INTEGRATIVA DE LITERATURA*
}

Paula Bresolin', Jussara Gue Martini², Daniele Delacanal Lazzari ${ }^{3}$, Isis da Silva Galindo ${ }^{4}$, Jeferson Rodrigues ${ }^{5}$, Maria Helena Pires Araújo Barbosa ${ }^{6}$

\section{RESUMO}

Objetivo: este estudo tem como objetivo conhecer as contribuições da aprendizagem experiencial para a conquista das competências gerais previstas nas Diretrizes Curriculares Nacionais de Enfermagem.

Método: realizou-se revisão integrativa, no período de janeiro de 2013 a agosto de 2017.

Resultados: para sistematizar a discussão dos resultados, os 43 artigos selecionados foram apresentados a partir das competências gerais definidas nas Diretrizes Curriculares Nacionais.

Conclusão: Evidenciou-se que a aprendizagem experiencial como estratégia de ensino e aprendizagem na enfermagem e suas contribuições alcançam as competências e habilidades gerais definidas pelas Diretrizes Curriculares Nacionais. A habilidade de Atenção à saúde foi a mais abordada nos estudos. Ressalta-se que a aprendizagem experiencial é potencializada quando atinge pensamento crítico-reflexivo na formação profissional. A simulação, abordada nos artigos selecionados, foi a metodologia efetiva de ensino para estudantes de enfermagem.

DESCRITORES: Educação em Enfermagem, Educação Baseada em Competências, Enfermagem, Ensino, Aprendizagem.

*Artigo extraído da dissertação de mestrado "Estilo Individual de Aprendizagem e a Simulação Clínica no Ensino Superior Em Enfermagem". Universidade Federal de Santa Catarina, 2018.

COMO REFERENCIAR ESTE ARTIGO:

Bresolin P, Martini JG, Lazzari DD, Galindo I da S, Rodrigues J, Barbosa MHPA. Aprendizagem experiencial e diretrizes curriculares nacionais de enfermagem: revisão integrativa de literatura (2013-2017). Cogitare enferm. [Internet]. 2019 [acesso em "colocar data de acesso, dia, mês abreviado e ano"]; 24. Disponível em: http:// dx.doi.org/10.5380/ce.v24i0.59024.

Este obra está licenciado com uma Licença Creative Commons Atribuição 4.0 Internacional.

${ }^{1}$ Enfermeira. Mestra em Enfermagem. Universidade Federal de Santa Catarina. Florianópolis, SC, Brasil. 0

${ }^{2}$ Enfermeira. Doutora em Enfermagem. Docente de Enfermagem da Universidade Federal de Santa Catarina. Florianópolis, SC, Brasil.

${ }^{3}$ Enfermeira. Doutora em Enfermagem Docente de Enfermagem da Universidade Federal de Santa Catarina. Florianópolis, SC, Brasil. 0

${ }^{4}$ Enfermeira, Mestra em Enfermagem. Universidade Federal de Santa Catarina. Florianópolis, SC, Brasil. $\odot$

${ }^{5}$ Enfermeiro. Doutor em Enfermagem. Docente de Enfermagem da Universidade Federal de Santa Catarina. Florianópolis, SC, Brasil.

${ }^{6}$ Enfermeira. Doutoranda em Enfermagem. Docente da Universidade Federal do Pará. Altamira, PA, Brasil. 9 


\title{
EXPERIENTIAL LEARNING AND NATIONAL CURRICULUM GUIDELINES FOR NURSING UNDERGRADUATE COURSES: INTEGRATIVE REVIEW OF THE LITERATURE
}

\begin{abstract}
Objective: The present study aims to gain insight on the contributions of experiential learning to the achievement of the general competencies provided for in the National Curriculum Guidelines for Nursing Undergraduate Courses.

Method: An integrative review was conducted from January 2013 to August 2017.

Results: For the systematization of the discussion of the results, the presentation of the 43 selected articles was based on the general competencies set out in the National Curriculum Guidelines.

Conclusion: Experiential learning as a teaching and learning strategy in nursing and its contributions are consistent with the general competencies and skills set out in the National Curriculum Guidelines. The Health Care skill was the most covered skill in all the studies. It is noteworthy that experiential learning is maximized when it reaches critical-reflective thinking in vocational training. The simulation addressed in the selected articles was an effective teaching methodology for nursing students.
\end{abstract}

DESCRIPTORS: Nursing Education, Competency-Based Education, Nursing, Teaching, Learning.

\section{APRENDIZAJE EXPERIENCIAL Y DIRECTRICES CURRICULARES NACIONALES DE ENFERMERIA: REVISIÓN INTEGRATIVA DE LITERATURA}

\begin{abstract}
RESUMEN:
Objetivo: El objetivo del estudio apunta a conocer los aportes del aprendizaje experiencial a la consecución de las competencias generales previstas en las Directrices Curriculares Nacionales de Enfermería.

Método: Se efectuó una revisión integrativa de literatura, entre enero de 2013 y agosto de 2017.

Resultados: Para sistematizar la discusión de los resultados, los 43 artículos seleccionados fueron presentados en función de las competencias generales definidas en las Directrices Curriculares Nacionales.

Conclusión: Se evidenció que el aprendizaje experiencial, como estrategia de enseñanza y aprendizaje en enfermería y sus contribuciones, satisfacen las competencias y habilidades generales definidas por las Directrices Curriculares Nacionales. La habilidad de Atención de Salud fue la más abordada en los estudios. Se resalta que el aprendizaje experiencial se potencia cuanto propicia el pensamiento crítico-reflexivo en la formación profesional. La simulación, abordada en los artículos seleccionados, fue la metodología de enseñanza más efectiva para los estudiantes de enfermería.
\end{abstract}

DESCRIPTORES: Educación en Enfermería; Educación Basada en Competencias; Enfermería; Enseñanza; Aprendizaje. 
Ao longo da trajetória histórica da educação superior no Brasil, em particular na área da saúde, foi possível observar mudanças significativas com a finalidade de acompanhar as transformações ocorridas na sociedade brasileira. Para acompanhar este processo, foi necessário um esforço conjunto do Ministério da Saúde e do Ministério da Educação, para criar as Diretrizes Curriculares Nacionais dos Cursos de Graduação (DCN).

Especificamente para o curso de Enfermagem, as DCN preconizam os princípios, fundamentos, condições e procedimentos da formação profissional e descrevem as competências e habilidades próprias do enfermeiro direcionadas à atenção à saúde, de modo a proporcionar ações de prevenção, promoção, proteção e reabilitação da saúde. Explicitam que a formação do enfermeiro tem por objetivo dotar esse profissional de conhecimentos necessários para o exercício das seguintes competências e habilidades gerais: atenção à saúde, tomada de decisões, comunicação, liderança e educação permanente ${ }^{(1)}$.

Para além, espera-se que o estudante ou futuro profissional seja capaz de auxiliar na transformação das condições precárias da saúde da população brasileira, posicionando-se criticamente frente ao contexto sócio-político-econômico do país, atuando como agente de mudança, no sentido de reconhecer o significado da prática de enfermagem no contexto social|(1).

O ensino da enfermagem comporta as vertentes educativas que incluem a vertente teórica, a teórico-prática e o ensino clínico(2). Neste processo de formação, é necessário considerar tanto o estudante de graduação quanto o trabalhador como um sujeito de aprendizagem, com o intuito de contribuir para um sistema de saúde vigente no país ${ }^{(3)}$.

Considerando esse contexto, os métodos de ensino devem alcançar o perfil acadêmico e profissional desejado e por esta razão procura-se compreender e aprimorar o processo de ensino e aprendizagem na busca de estratégias pedagógicas que abarquem as diferentes formas de aprender, considerando as individualidades e características de aprendizagem ${ }^{(4)}$.

O teórico David A. Kolb direciona-se ao conhecimento de como se apreende e se assimila informação, como se solucionam problemas e se tomam decisões, e esses questionamentos levaram-no a elaborar um modelo que denominou experiencial, com o qual busca conhecer o processo da aprendizagem baseada na própria experiência. Uma de suas primeiras afirmações é derivada deste primeiro argumento: "se as organizações e instituições considerassem o modelo de como os indivíduos aprendem, estas estariam em condiçỗes de melhorar e aumentar a capacidade de aprender" (5).

De acordo com Kolb, há um ciclo com quatro estratégias de aprendizagem. A Experiência Concreta enfatiza as experiências pessoais e os sentimentos envolvidos na situação de aprendizagem. Na Observação Reflexiva, o aprendiz acredita poder resolver seus problemas usando pensamentos e sentimentos e dando ênfase à paciência, julgamentos cuidadosos e habilidade para entender ideias sob diversos pontos de vista. $\mathrm{Na}$ Conceituação Abstrata, o entendimento é baseado na compreensão intelectual de uma situação, e o nível de abstração é elevado. Por fim, a Experimentação Ativa, que envolve a aprendizagem de forma ativa. Desta forma, o Ciclo de Aprendizagem de Kolb é uma ferramenta que utiliza experiências para contribuir no desenvolvimento do indivíduo, uma vez que apresentam um objetivo específico para com a aprendizagem ${ }^{(6)}$.

Esta pesquisa teve como objetivo conhecer as contribuições da aprendizagem experiencial para a conquista das competências e habilidades próprias do enfermeiro direcionadas à atenção à saúde previstas nas DCN de Enfermagem. 
Adotou-se a revisão integrativa da literatura como método, constituída por seis etapas: identificação do problema, estabelecimento de critérios de inclusão/exclusão (seleção da amostra), definição das informações a serem extraídas dos artigos selecionados, análise das informações, interpretação dos resultados e apresentação da revisão(7).

A pesquisa teve início após consultas às bases de dados BVS (BIREME - Biblioteca Virtual em Saúde), PubMed NCBI, Scopus, Web of Science e ERIC (Education Resources Information Center), com base na questão norteadora: quais as contribuições da aprendizagem experiencial para a educação em enfermagem? Os critérios para inclusão foram artigos disponíveis eletronicamente na íntegra nos idioma português, espanhol e inglês, publicados no período de janeiro de 2013 a agosto de 2017. Excluíram-se editoriais, cartas, artigos de opinião, comentários e notas prévias, bem como as publicações duplicadas em mais de uma base de dados, teses, dissertações e manuais.

A análise e interpretação dos dados foram realizadas após tradução e leitura dos artigos, por meio das seguintes etapas: 1) identificação da hipótese ou questão norteadora - elaboração de uma problemática pelo pesquisador de maneira clara e objetiva, seguida da busca pelos descritores e/ou palavras-chaves; 2) seleção da amostragem determinação dos critérios de inclusão ou exclusão, estabelecendo a transparência para que proporcione profundidade, qualidade e confiabilidade na seleção; 3) categorização dos estudos - definição quanto à extração das informações dos artigos revisados com o objetivo de sumarizá-las e organizá-las; 4) avaliação dos estudos - análise crítica dos dados extraídos; 5) discussão e interpretação dos resultados - comparação e fundamentação dos principais resultados com o conhecimento teórico e avaliação quanto a sua aplicabilidade; 6) apresentação da revisão integrativa e síntese do conhecimento - informações de cada artigo revisado de maneira sucinta e sistematizadas, demonstrando as evidências encontradas ${ }^{(7)}$.

A coleta de dados foi desenvolvida no mês de agosto de 2017. Os materiais extraídos das bases de dados foram exportados para o EndNote: ano de publicação, título, autores, periódico, país de origem do estudo, tipo/abordagem do estudo e principais aspectos ou resultados. Quanto aos aspectos éticos, foram respeitados os direitos autorais e o conteúdo, não havendo modificação destes em benefício da revisão.

A estratégia de busca ocorreu nas bases de dados com as palavras-chave e/ou descritores: "Pupil Nurses" OR "Student, Nursing" OR "Nurses, Pupil" OR "Nurse, Pupil" $O R$ "Pupil Nurse" OR "Nursing Student" OR "Nursing Students" OR "Nursing Education" $O R$ "Educations, Nursing" OR "Nursing Educations" Or nursing AND "Problem-Based Learning" OR "Experiential Learning" OR "Learning, Experiential" OR Kolb OR "David Kolb".

Esta busca resultou em 2.315 artigos. Destes, 980 encontravam-se repetidos, 772 apresentavam outro método de aprendizagem, 223 não envolviam estudos na enfermagem, 233 não explicitaram metodologias de aprendizagem, 11 eram editoriais e 53 não permitiam acesso ao artigo na íntegra. Restaram 43 artigos, conforme a Tabela 1, que foram posteriormente discutidos considerando as competências e habilidades gerais contidas nas DCN de Enfermagem.

Tabela 1 - Publicações distribuídas por quantitativo, segundo bases eletrônicas consultadas. Florianópolis, SC, Brasil, 2017

Número de artigos encontrados
Número de Artigos selecionados 


\begin{tabular}{lll}
\hline ERIC & 20 & 1 \\
\hline PUBMED & 677 & 8 \\
\hline SCOPUS & 525 & 9 \\
\hline WEB OF SCIENCE & 229 & 9 \\
\hline Total & 2315 & 43
\end{tabular}

\section{RESULTADOS}

Dos 43 artigos, 36 apontam questões relacionadas à atenção à saúde, 11 com a tomada de decisão, 18 abordam aspectos de comunicação, nove tratam da liderança, quatro discutem a formação para ações de administração e gerenciamento e 17 educação permanente. Na sequência, abordamos os elementos apresentados pelos artigos analisados em cada uma das categorias. As categorias consideradas para este item são: atenção à saúde, tomada de decisões, comunicação, liderança e educação permanente.

O Quadro 1 sintetiza as informações concernentes aos 43 artigos selecionados para este estudo, incluindo título, autores, ano de publicação e local, tipo do estudo e participantes.

Quadro 1 - Publicações distribuídas por variável de análise. Florianópolis, SC, Brasil, 2017 (continua)

\begin{tabular}{|l|l|l|l|}
\hline Título & Autores/Ano/Local & $\begin{array}{l}\text { Tipo de } \\
\text { Estudo }\end{array}$ & Participantes \\
\hline $\begin{array}{l}\text { The evaluation of simulation market in } \\
\text { nursing education and the determination } \\
\text { of learning style of students }\end{array}$ & $\begin{array}{l}\text { Celik Y, Ceylantekin Y, Kilic I. } \\
2017 \\
\text { Turquia }\end{array}$ & $\begin{array}{l}\text { Quantitativo } \\
\text { Descritivo }\end{array}$ & $\begin{array}{l}\text { Estudantes de } \\
\text { Enfermagem }\end{array}$ \\
\hline $\begin{array}{l}\text { Ethical experiential learning in medical, } \\
\text { nursing and allied health education: a } \\
\text { narrative review }\end{array}$ & $\begin{array}{l}\text { Grace S, Innes E, Patton N, } \\
\text { Stockhausen L. } \\
2017 \\
\text { Austrália }\end{array}$ & $\begin{array}{l}\text { Revisão } \\
\text { narrativa de } \\
\text { literatura }\end{array}$ & $\begin{array}{l}\text { Artigos de } \\
2000 \text { a } 2015\end{array}$ \\
\hline $\begin{array}{l}\text { Nursing students' experiential learning } \\
\text { processes using an online 3D simulation } \\
\text { game }\end{array}$ & $\begin{array}{l}\text { Koivisto J-M, Niemi H, } \\
\text { Multisilta J, Eriksson E. } \\
2017 \\
\text { Finlândia }\end{array}$ & $\begin{array}{l}\text { Qualitativo } \\
\text { - entrevistas } \\
\text { com grupo } \\
\text { focal }\end{array}$ & $\begin{array}{l}\text { Estudantes de } \\
\text { Enfermagem }\end{array}$ \\
\hline $\begin{array}{l}\text { Analysis of student reflections of } \\
\text { experiential learning in nursing health } \\
\text { policy courses }\end{array}$ & $\begin{array}{l}\text { McGuire M, Goldstein C, } \\
\text { Claywell L, Patton R. } \\
2017 \\
\text { EUA }\end{array}$ & $\begin{array}{l}\text { Qualitativo - } \\
\text { retrospectivo }\end{array}$ & $\begin{array}{l}\text { Mestrandos e } \\
\text { doutorandos } \\
\text { de } \\
\text { enfermagem }\end{array}$ \\
\hline $\begin{array}{l}\text { Implementation of a state legislative } \\
\text { fellowship for doctor of nursing practice } \\
\text { students }\end{array}$ & $\begin{array}{l}\text { Crowder SJ, Ironside PM, } \\
\text { Cangany M, Roddy WS. } \\
2016 \\
\text { EUA }\end{array}$ & $\begin{array}{l}\text { Relato de } \\
\text { experiência }\end{array}$ & $\begin{array}{l}\text { Doutores de } \\
\text { Enfermagem }\end{array}$ \\
\hline $\begin{array}{l}\text { Perceptions of graduating health } \\
\text { professional students of their } \\
\text { interprofessional education experiences } \\
\text { during pre-licensure education }\end{array}$ & $\begin{array}{l}\text { Doucet S, Loney E, Brown } \\
\text { PA. } \\
2016 \\
\text { Canadá }\end{array}$ & $\begin{array}{l}\text { Qualitativo } \\
\text { Descritivo }\end{array}$ & $\begin{array}{l}\text { Estudantes de } \\
\text { Enfermagem, } \\
\text { Medicina, } \\
\text { Farmácia e } \\
\text { Fisioterapia }\end{array}$ \\
\hline
\end{tabular}




\begin{tabular}{|c|c|c|c|}
\hline $\begin{array}{l}\text { The impact of a simulated intervention } \\
\text { on attitudes of undergraduate nursing } \\
\text { and medical students towards end of life } \\
\text { care provision }\end{array}$ & $\begin{array}{l}\text { Lewis C, Reid J, McLernon } \\
\text { Z, Ingham R, Traynor M. } \\
2016 \\
\text { Reino Unido }\end{array}$ & $\begin{array}{l}\text { Quantitativo } \\
\text { Quase } \\
\text { experimental }\end{array}$ & $\begin{array}{l}\text { Estudantes de } \\
\text { Enfermagem }\end{array}$ \\
\hline $\begin{array}{l}\text { Service learning in undergraduate } \\
\text { nursing } \\
\text { education: strategies to facilitate } \\
\text { meaningful reflection }\end{array}$ & $\begin{array}{l}\text { Schmidt NA, Brown JM. } \\
2016 \\
\text { Chile }\end{array}$ & Reflexão & $\begin{array}{l}\text { Estudantes de } \\
\text { Enfermagem }\end{array}$ \\
\hline $\begin{array}{l}\text { New ways of seeing: Nursing students } \\
\text { experiences of a pilot service learning } \\
\text { program in Australia. }\end{array}$ & $\begin{array}{l}\text { Townsend L, Gray J, Forber } \\
\text { J. } \\
2016 \\
\text { Austrália }\end{array}$ & $\begin{array}{l}\text { Qualitativo. } \\
\text { Descritivo }\end{array}$ & $\begin{array}{l}\text { Estudantes de } \\
\text { Enfermagem }\end{array}$ \\
\hline $\begin{array}{l}\text { Sculpting with people - An experiential } \\
\text { learning technique }\end{array}$ & $\begin{array}{l}\text { Andersen HE, Larsen KV. } \\
2015 \\
\text { Dinamarca }\end{array}$ & $\begin{array}{l}\text { Qualitativo. } \\
\text { Descritivo }\end{array}$ & $\begin{array}{l}\text { Estudantes de } \\
\text { Enfermagem }\end{array}$ \\
\hline $\begin{array}{l}\text { The use and application of drama in } \\
\text { nursing education - an integrative review } \\
\text { of the literature }\end{array}$ & $\begin{array}{l}\text { Arveklev SH, Wigert H, Berg } \\
\text { L, Burton B, Lepp M. } \\
2015 \\
\text { Dinamarca }\end{array}$ & $\begin{array}{l}\text { Qualitativo. } \\
\text { Revisão } \\
\text { integrativa }\end{array}$ & $\begin{array}{l}\text { Artigos de } \\
2003 \text { a } 2014 .\end{array}$ \\
\hline $\begin{array}{l}\text { Experiential learning: using virtual } \\
\text { simulation in an online } R N \text {-to-BSN } \\
\text { program }\end{array}$ & $\begin{array}{l}\text { Breen } \mathrm{H} \text {, Jones } \mathrm{M} . \\
2015 \\
\text { EUA }\end{array}$ & Qualitativo & $\begin{array}{l}\text { Estudantes de } \\
\text { Enfermagem }\end{array}$ \\
\hline $\begin{array}{l}\text { Effects of an experiential learning } \\
\text { simulation design on clinical nursing } \\
\text { judgment development }\end{array}$ & $\begin{array}{l}\text { Chmil JV, Turk M, Adamson } \\
\text { K, Larew C. } \\
2015 \\
\text { EUA }\end{array}$ & $\begin{array}{l}\text { Quantitativo. } \\
\text { Quase- } \\
\text { Experimental }\end{array}$ & $\begin{array}{l}\text { Estudantes de } \\
\text { Enfermagem }\end{array}$ \\
\hline $\begin{array}{l}\text { Experiential learning in nursing } \\
\text { consultation education via clinical } \\
\text { simulation with actors: action research }\end{array}$ & $\begin{array}{l}\text { De Oliveira SN, Prado } \\
\text { MLD, Kempfer SS, Martini } \\
\text { JG, Caravaca-Morera JA, } \\
\text { Bernardi MC. } 2015 \\
\text { Brasil } \\
\end{array}$ & Pesquisa-Ação & $\begin{array}{l}\text { Estudantes de } \\
\text { Enfermagem }\end{array}$ \\
\hline $\begin{array}{l}\text { Vaccination learning experiences of } \\
\text { nursing students: a grounded theory } \\
\text { study }\end{array}$ & $\begin{array}{l}\text { Ildarabadi E, Karimi } \\
\text { Moonaghi H, Heydari } \\
\text { A,Taghipour A, } \\
\text { Abdollahimohammad A. } \\
2015 \\
\text { Irã } \\
\end{array}$ & $\begin{array}{l}\text { Qualitativo. } \\
\text { Teoria } \\
\text { Fundamentada } \\
\text { nos Dados } \\
\text { (TFD) }\end{array}$ & $\begin{array}{l}\text { Estudantes de } \\
\text { Enfermagem }\end{array}$ \\
\hline $\begin{array}{l}\text { Development of a post-simulation } \\
\text { debriefing intervention to prepare } \\
\text { nurses and nursing students to care for } \\
\text { deteriorating patients }\end{array}$ & $\begin{array}{l}\text { Lavoie P, Pepin J, Cossette } \\
\text { S. } \\
2015 \\
\text { Canadá }\end{array}$ & $\begin{array}{l}\text { Revisão de } \\
\text { Literatura }\end{array}$ & $\begin{array}{l}\text { Artigos } 2002 \\
\text { a } 2012 .\end{array}$ \\
\hline $\begin{array}{l}\text { Enhancing empathy in undergraduate } \\
\text { nursing students: An experiential } \\
\text { ostomate simulation }\end{array}$ & $\begin{array}{l}\text { Maruca AT, Díaz DA, Kuhnly } \\
\text { JE, Jeffries PR. } \\
2015 \\
\text { EUA } \\
\end{array}$ & $\begin{array}{l}\text { Quantitativo. } \\
\text { Experimental }\end{array}$ & $\begin{array}{l}\text { Estudantes de } \\
\text { Enfermagem }\end{array}$ \\
\hline $\begin{array}{l}\text { Preparing students for clinical } \\
\text { placements: The student's perspective. }\end{array}$ & $\begin{array}{l}\text { McNamara N. } \\
2015 \\
\text { Nova Zelândia } \\
\end{array}$ & $\begin{array}{l}\text { Qualitativo. } \\
\text { Quantitativo. } \\
\text { Descritivo }\end{array}$ & $\begin{array}{l}\text { Estudantes de } \\
\text { Enfermagem }\end{array}$ \\
\hline $\begin{array}{l}\text { How learning styles and preferences of } \\
\text { first-year nursing and midwifery students } \\
\text { change }\end{array}$ & $\begin{array}{l}\text { Mitchell EKL, James S, } \\
\text { D'Amore A. } \\
2015 \\
\text { Austrália }\end{array}$ & $\begin{array}{l}\text { Quantitativo } \\
\text { Transversal }\end{array}$ & $\begin{array}{l}\text { Estudantes de } \\
\text { Enfermagem }\end{array}$ \\
\hline $\begin{array}{l}\text { A pedagogia por trás da experiência } \\
\text { clínica simulada: uma percepção de }\end{array}$ & $\begin{array}{l}\text { Nunes de Oliveira S, do } \\
\text { Prado ML, Silveira Kempfer }\end{array}$ & $\begin{array}{l}\text { Qualitativo } \\
\text { Pesquisa-Ação }\end{array}$ & $\begin{array}{l}\text { Estudantes de } \\
\text { Enfermagem }\end{array}$ \\
\hline
\end{tabular}




\begin{tabular}{|c|c|c|c|}
\hline estudantes de enfermagem & $\begin{array}{l}\text { S, Waterkemper R, Caravaca } \\
\text { Morera JA, Bernardi MC. } \\
2015 \\
\text { Brasil } \\
\end{array}$ & & \\
\hline $\begin{array}{l}\text { La enseñanza de la enfermeira con } \\
\text { simuladores, consideraciones teórico- } \\
\text { pedagógicas para perfilar un modelo } \\
\text { didáctico }\end{array}$ & $\begin{array}{l}\text { Piña-Jiménez l, Amador- } \\
\text { Aguilar R. } \\
2015 \\
\text { México } \\
\end{array}$ & Reflexão & \\
\hline $\begin{array}{l}\text { Identification of the learning styles } \\
\text { and "On-the-Job" learning methods } \\
\text { implemented by nurses for promoting } \\
\text { their professional knowledge and skills }\end{array}$ & $\begin{array}{l}\text { Rassin M, Kurzweil Y, Maoz } \\
\text { Y. } \\
2015 \\
\text { Israel }\end{array}$ & $\begin{array}{l}\text { Quantitativo } \\
\text { Descritivo }\end{array}$ & Enfermeiros \\
\hline $\begin{array}{l}\text { Learning style impact on knowledge } \\
\text { gains in human patient simulation }\end{array}$ & $\begin{array}{l}\text { Shinnick MA, Woo MA. } \\
2015 \\
\text { EUA }\end{array}$ & $\begin{array}{l}\text { Quantitativo } \\
\text { Estudo de } \\
\text { Coorte }\end{array}$ & $\begin{array}{l}\text { Estudantes de } \\
\text { Enfermagem }\end{array}$ \\
\hline $\begin{array}{l}\text { Experiential learning not enough for } \\
\text { organ procurement surgery: implications } \\
\text { for perioperative nursing education }\end{array}$ & $\begin{array}{l}\text { Smith Z, Leslie G, Wynaden } \\
\text { D. } 2015 \\
\text { Austrália } \\
\end{array}$ & $\begin{array}{l}\text { Qualitativo } \\
\text { TFD }\end{array}$ & Enfermeiros \\
\hline $\begin{array}{l}\text { Learning end-of-life care within a } \\
\text { constructivist model: Undergraduate } \\
\text { nursing students' experiences }\end{array}$ & $\begin{array}{l}\text { Van der Wath AE, Du Toit } \\
\text { PH. } \\
2015 \\
\text { África do Sul }\end{array}$ & $\begin{array}{l}\text { Qualitativo } \\
\text { Descritivo }\end{array}$ & $\begin{array}{l}\text { Estudantes de } \\
\text { Enfermagem }\end{array}$ \\
\hline $\begin{array}{l}\text { Utilization of experiential learning, and } \\
\text { the learning outcomes model reduces } \\
\text { RN orientation time by more than } 35 \% \text {. }\end{array}$ & $\begin{array}{l}\text { Zigmont JJ, Wade A, } \\
\text { Edwards T, Hayes K, } \\
\text { Mitchell J, Oocumma N. } \\
2015 \\
\text { EUA }\end{array}$ & $\begin{array}{l}\text { Qualitativo } \\
\text { Quantitativo }\end{array}$ & Enfermeiros. \\
\hline $\begin{array}{l}\text { An evaluation of advanced simulation in } \\
\text { nursing: a mixed-method study }\end{array}$ & $\begin{array}{l}\text { Chiang VC, Chan SS. } \\
2014 \\
\text { China }\end{array}$ & $\begin{array}{l}\text { Qualitativo } \\
\text { Quantitativo }\end{array}$ & $\begin{array}{l}\text { Estudantes de } \\
\text { Enfermagem }\end{array}$ \\
\hline Teaching delegation to RN students. & $\begin{array}{l}\text { Dai L, } \\
\text { Turner K, Anderson RA, } \\
\text { Mueller C, McConnell ES, } \\
\text { Corazzini KN2014 } \\
\text { EUA } \\
\end{array}$ & Estudo de caso & $\begin{array}{l}\text { Estudantes de } \\
\text { Enfermagem }\end{array}$ \\
\hline $\begin{array}{l}\text { An evaluation of mental health } \\
\text { simulation with standardized patients }\end{array}$ & $\begin{array}{l}\text { Doolen J, Giddings M, } \\
\text { Johnson M, Guizado de } \\
\text { Nathan G, O Badia L. } \\
2014 \\
\text { EUA }\end{array}$ & Estudo de caso & $\begin{array}{l}\text { Estudantes de } \\
\text { Enfermagem }\end{array}$ \\
\hline $\begin{array}{l}\text { The role of critical thinking skills and } \\
\text { learning styles of university students in } \\
\text { their academic performance }\end{array}$ & $\begin{array}{l}\text { Ghazivakili Z, Norouzi } \\
\text { Nia R, Panahi F, Karimi M, } \\
\text { Gholsorkhi H, Ahmadi Z. } \\
2014 \\
\text { EUA }\end{array}$ & $\begin{array}{l}\text { Quantitativo } \\
\text { Estudo de } \\
\text { correlação } \\
\text { cruzada. }\end{array}$ & $\begin{array}{l}\text { Estudantes de } \\
\text { Enfermagem, } \\
\text { Medicina, } \\
\text { Obstetrícia } \\
\text { e Saúde e } \\
\text { Emergência } \\
\text { Médica } \\
\end{array}$ \\
\hline $\begin{array}{l}\text { Nursing students' experiences of } \\
\text { involvement in clinical research: an } \\
\text { exploratory study }\end{array}$ & $\begin{array}{l}\text { Henoch I, Jakobsson Ung } \\
\text { E, Ozanne A, Falk H, Falk K, } \\
\text { Kenne Sarenmalm E, et al. } \\
2014 \\
\text { Suécia }\end{array}$ & $\begin{array}{l}\text { Quantitativo } \\
\text { Qualitativo. } \\
\text { Descritivo } \\
\text { Transversal }\end{array}$ & $\begin{array}{l}\text { Estudantes de } \\
\text { Enfermagem }\end{array}$ \\
\hline $\begin{array}{l}\text { Engaging the learner by bridging } \\
\text { the gap between theory and clinical } \\
\text { competence: the impact of concept }\end{array}$ & $\begin{array}{l}\text { Jamison T, Lis GA. } \\
2014 \\
\text { EUA }\end{array}$ & Reflexão & $\begin{array}{l}\text { Estudantes de } \\
\text { Enfermagem } \\
\text { e Enfermeiros. }\end{array}$ \\
\hline
\end{tabular}




\begin{tabular}{|c|c|c|c|}
\hline $\begin{array}{l}\text { mapping and simulation as innovative } \\
\text { strategies for nurse-sensitive outcome } \\
\text { indicators }\end{array}$ & & & \\
\hline $\begin{array}{l}\text { Mock board hearing: giving students } \\
\text { new insights through experiential } \\
\text { learning }\end{array}$ & $\begin{array}{l}\text { Jordan SD, Collins-Yoder } \\
\text { AS. } \\
2014 \\
\text { EUA }\end{array}$ & $\begin{array}{l}\text { Relato de } \\
\text { Experiência }\end{array}$ & $\begin{array}{l}\text { Estudantes de } \\
\text { Enfermagem }\end{array}$ \\
\hline $\begin{array}{l}\text { Development and evaluation of a } \\
\text { learner-centered educational summer } \\
\text { camp program on soft skills for } \\
\text { baccalaureate nursing students. }\end{array}$ & $\begin{array}{l}\text { Lau Y, Wang W. } \\
2014 \\
\text { China }\end{array}$ & $\begin{array}{l}\text { Qualitativo } \\
\text { Quantitativo } \\
\text { Grupo Focal }\end{array}$ & $\begin{array}{l}\text { Estudantes de } \\
\text { Enfermagem }\end{array}$ \\
\hline $\begin{array}{l}\text { Simulation-based interprofessional } \\
\text { education guided by Kolb's experiential } \\
\text { learning theory }\end{array}$ & $\begin{array}{l}\text { Poore JA, Cullen DL, Schaar } \\
\text { GL. } 2014 \\
\text { EUA }\end{array}$ & $\begin{array}{l}\text { Revisão de } \\
\text { Literatura }\end{array}$ & $\begin{array}{l}\text { Estudantes de } \\
\text { Enfermagem }\end{array}$ \\
\hline $\begin{array}{l}\text { A virtual pregnancy for pre-licensure } \\
\text { nursing students: nine months up and } \\
\text { close }\end{array}$ & $\begin{array}{l}\text { Weideman YL, Culleiton AL. } \\
2014 \\
\text { EUA }\end{array}$ & Qualitativo & $\begin{array}{l}\text { Estudantes de } \\
\text { Enfermagem }\end{array}$ \\
\hline $\begin{array}{l}\text { Self-directed learning readiness } \\
\text { and learning styles among Saudi } \\
\text { undergraduate nursing students }\end{array}$ & $\begin{array}{l}\text { El-Gilany A-H, Abusaad FES. } \\
2013 \\
\text { Arábia Saudita }\end{array}$ & $\begin{array}{l}\text { Quantitativo } \\
\text { Descritivo } \\
\text { Transversal } \\
\end{array}$ & $\begin{array}{l}\text { Estudantes de } \\
\text { Enfermagem }\end{array}$ \\
\hline $\begin{array}{l}\text { The perceived benefits of a virtual } \\
\text { community effects of learning style, } \\
\text { race, ethnicity, and frequency of use on } \\
\text { nursing students. }\end{array}$ & $\begin{array}{l}\text { Fogg L, Carlson-Sabelli L, } \\
\text { Carlson K, Giddens J. } 2013 \\
\text { EUA }\end{array}$ & Qualitativo & $\begin{array}{l}\text { Estudantes de } \\
\text { Enfermagem }\end{array}$ \\
\hline $\begin{array}{l}\text { Nursing student anxiety as a context for } \\
\text { teaching/learning. }\end{array}$ & $\begin{array}{l}\text { Hutchinson TL, Janiszewski } \\
\text { Goodin H. } \\
2013 \\
\text { EUA }\end{array}$ & Reflexão & $\begin{array}{l}\text { Estudantes de } \\
\text { Enfermagem }\end{array}$ \\
\hline $\begin{array}{l}\text { Improving collaboration among medical, } \\
\text { nursing and respiratory therapy students } \\
\text { through interprofessional simulation. }\end{array}$ & $\begin{array}{l}\text { King AEA, Conrad M, } \\
\text { Ahmed RA. } 2013 \\
\text { EUA }\end{array}$ & Qualitativo & $\begin{array}{l}\text { Estudantes de } \\
\text { Enfermagem } \\
\text { e Medicina } \\
\end{array}$ \\
\hline $\begin{array}{l}\text { Nursing student voices: reflections on an } \\
\text { international service learning experience. }\end{array}$ & $\begin{array}{l}\text { Main EE, Garrett-Wright D, } \\
\text { Kerby M. } \\
2013 \\
\text { Austrália }\end{array}$ & Qualitativo & $\begin{array}{l}\text { Estudantes } \\
\text { de graduação } \\
\text { e pós } \\
\text { graduação em } \\
\text { enfermagem }\end{array}$ \\
\hline $\begin{array}{l}\text { A novel approach to improve hand } \\
\text { hygiene compliance of student nurses. }\end{array}$ & $\begin{array}{l}\text { Salmon S, Wang XB, Seetoh } \\
\text { T, Lee SY, Fisher DA. } \\
2013 \\
\text { Singapura }\end{array}$ & $\begin{array}{l}\text { Quantitativo } \\
\text { Exploratório }\end{array}$ & $\begin{array}{l}\text { Estudantes de } \\
\text { Enfermagem }\end{array}$ \\
\hline $\begin{array}{l}\text { An innovative tool for experiential } \\
\text { learning of nursing quality and safety } \\
\text { competencies }\end{array}$ & $\begin{array}{l}\text { St Onge J, Hodges T, } \\
\text { McBride M, Parnell R. } \\
2013 \\
\text { EUA }\end{array}$ & $\begin{array}{l}\text { Relato de } \\
\text { experiência }\end{array}$ & $\begin{array}{l}\text { Estudantes de } \\
\text { Enfermagem }\end{array}$ \\
\hline
\end{tabular}

Quanto ao ano de publicação, a expressividade numérica está em 2015, com 17 artigos publicados, no ano anterior (2014) aparecem 10 artigos publicados e 2013, sete artigos. Percebeu-se diminuição na atualidade, pois com relação aos anos de 2017 e 2016 foram encontrados respectivamente quatro e cinco artigos publicados. Destaca-se a superioridade de produções apresentada pelos Estados Unidos com 18 artigos, seguido 
pela Austrália com cinco. As outras 20 publicações inseridas neste estudo são referentes a 16 países.

A maioria das publicações não foi produzida no Brasil, no entanto os artigos examinados abordam questões que podem ser relacionadas com a formação nos cursos brasileiros, trazendo aspectos semelhantes aos apresentados nas DCN.

\section{DISCUSSÃO}

\section{Atenção à saúde}

Os profissionais de enfermagem devem ser condicionados a processos formativos que direcionem o pensar e agir criticamente, tanto no nível individual como coletivo em uma determinada sociedade ${ }^{(1)}$. Esses processos devem contribuir para a atuação profissional em consonância com as políticas públicas de saúde, dentre elas, a Política Nacional de Promoção a Saúde, cujo objetivo é a implantação de ações de promoção da saúde voltadas à comunidade, enfatizando a qualidade de vida, redução de vulnerabilidade e riscos à saúde da população com ênfase na atenção básica ${ }^{(8)}$.

A formação profissional voltada para a atenção à saúde desenvolve ações de prevenção, promoção, proteção e reabilitação da saúde, e foi abordada nos estudos em cuidados ao fim da vida(9-10), saúde mental(10-12), insuficiência cardíaca ${ }^{(13)}$, vacinação(14), exame

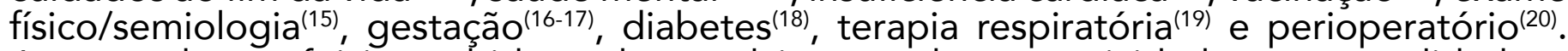
Aos estudantes foi incumbido o desenvolvimento de suas atividades com qualidade e dentro dos princípios da ética/bioética, enfatizando o desenvolvimento de aprendizagem e promovendo o trabalho interprofissional(19).

Uma das estratégias de ensino que proporcionou aos estudantes habilidades clínicas foi a simulação(18,21-24), que mostrou-se um método capaz de propiciar a aprendizagem experiencial ao recriar um cenário, provocando no estudante a curiosidade epistemológica, aproximando a experiência ao contexto teórico, transformando-a ${ }^{(16,18,22)}$. Este método de ensino concentra-se no estudante e oferece incremento no desenvolvimento de várias habilidades, favorecendo a existência do processo de ensino e aprendizagem em ambiente seguro, para com o estudante e com o paciente ${ }^{(25)}$.

A atenção à saúde demonstrou, por maior número de estudos, o ensinamento através da aprendizagem experiencial. No ensino, os currículos materializados em conteúdos e, propriamente, nas aulas teóricas, teórico-práticas e nos estágios curriculares, estão cada vez mais afinados ao modelo da atenção básica à saúde. São desafios oriundos do processo de aprendizagem dos docentes para com os estudantes, envolvendo o trabalho dos profissionais de serviços da atenção à saúde, que, ao vivenciarem as práticas por meio da aprendizagem experiencial, promovem o comprometimento e a responsabilidade de todos os agentes envolvidos na aprendizagem ${ }^{(26)}$.

\section{Tomada de decisões}

Nos artigos analisados, a tomada de decisão foi atingida por meio de duas vertentes, uma através do pensamento crítico ${ }^{(12,27-29)}$ e a outra com base nos julgamentos clínicos ${ }^{(30-35)}$. Os autores apontam que, para aprender a pensar criticamente, é necessário estimular o ato reflexivo, o que significa desenvolver a capacidade de observação, análise, crítica, autonomia de pensar e de ideias, ampliar os horizontes, tornar-se agente ativo nas transformações da sociedade, buscar interagir com a realidade ${ }^{(36)}$.

Um dos estudos realizou uma atividade de aprendizagem experiencial em raciocínio clínico, na qual os estudantes foram direcionados a usar o modelo de simulação virtual em um programa online para atender às necessidades de um membro da família de sua 
comunidade virtual, como oportunidade para demonstrar a tomada de decisão na definição de cuidados de enfermagem ${ }^{(12)}$. Outros estudos utilizaram a aprendizagem experiencial através de jogos online para a resolução de problemas e tomada de decisão(22,37). Os resultados dos estudos enfatizam ainda a busca de conhecimento baseado em evidências para melhor orientar a própria tomada de decisão na prática clínica ${ }^{(12,22,37)}$.

Em 2015, o Ministério da Saúde lançou a apostila Síntese de Evidências para Políticas de Saúde, estimulando o uso de evidências científicas na tomada de decisão. O objetivo foi melhorar a aplicação de recursos, buscando maior efetividade na promoção, prevenção e atenção à saúde. Na cartilha, justifica-se a pouca utilização das evidências científicas a diferentes fatores, desde dificuldades que tomadores de decisão têm para interpretar, adaptar e aplicar o conhecimento científico até o baixo nível de interação entre política e pesquisa. Deste modo, acrescentam que, para melhorar o uso das evidểncias científicas na formulação e implementação de políticas de saúde, deve-se inclui processos de tradução e disseminação do conhecimento, considerando sempre os contextos institucionais e sociais relacionados, além de aspectos de equidade ${ }^{(38)}$.

O julgamento clínico apresentado nos estudos ${ }^{(30-31)}$ foi influenciado pelas atitudes dos estudantes e profissionais, preconceitos, perspectiva filosófica e capacidade de pensar criticamente ${ }^{(22,27-28)}$. O raciocínio clínico requer que os indivíduos dominem habilidades de pensamento de ordem superior para que possam ser alcançadas através do desenvolvimento do pensamento crítico e reflexivo na atuação profissional de enfermagem ${ }^{(12,35)}$.

$\mathrm{Na}$ maior parte dos estudos desta categoria, os desafios foram lançados na estratégia de simulação ${ }^{(27,29,31-33,35)}$, role-play ${ }^{(22,34)}$, drama ${ }^{(28)}$ e comunidade virtual(12), através da aprendizagem experiencial, que ocorreu individualmente e com interações entre estudantes e profissionais, permitindo a construção, compartilhamento e discussão de casos clínicos, possibilitando a reflexão para tomada de decisão.

\section{Comunicação}

A comunicação possibilita interações entre os indivíduos, podendo ocorrer de forma verbal ou não verbal|(1). Para além de uma simples interação, a comunicação é elemento chave para a garantia da qualidade da atenção à saúde entre pessoas, famílias e comunidade. Nos artigos analisados neste estudo, a abordagem da comunicação compreendeu dois aspectos: comunicação interprofissional e comunicação entre estudantes/profissionais e indivíduos/famílias ${ }^{(9,15,39-40)}$.

A comunicação interprofissional está relacionada à interação entre profissionais e/ou estudantes de diferentes áreas. A comunicação intra e intergrupos é capaz de transformar os modos de relação e de comunicação entre os sujeitos implicados nos processos de produção de saúde ${ }^{(41)}$. Desta forma, a comunicação pode melhorar a atenção à saúde por evidenciar a necessidade da colaboração e da corresponsabilização( ${ }^{(42)}$.

As estratégias identificadas nos estudos para abordar a comunicação entre estudantes/profissionais e indivíduos/famílias, utilizando a aprendizagem experiencial, foram essencialmente: a simulação(18,25,43), a aprendizagem em serviço ${ }^{(14,21-22,35,40)}$ e o role play ${ }^{(22,34)}$. Tais estratégias de aprendizagem trouxeram benefícios como a melhoria da comunicação entre estudantes/profissionais e clientes e familiares; aumento da confiança dos familiares dos pacientes quanto às práticas de Enfermagem executadas por estudantes; superação do medo e da ansiedade no primeiro contato com clientes $\mathbf{s}^{(9,14,18,29,35,40)}$.

Sendo assim, diante do desafio da comunicação efetiva nos serviços de saúde, as estratégias da aprendizagem experiencial podem contribuir para a melhor formação do profissional de Enfermagem e, por consequência, para a melhoria das práticas de Enfermagem.

\section{Liderança}


A liderança é competência considerada indispensável à prática do profissional enfermeiro na sociedade, e é a partir dela que se dirige o processo de trabalho e se conduz a equipe ao alcance de objetivos comuns ${ }^{(44)}$. Conceituada nas DCN, a liderança está relacionada ao trabalho em equipe multiprofissional, destacando compromisso, responsabilidade, empatia, habilidade para tomada de decisões, comunicação e gerenciamento de forma eficaz e efetiva ${ }^{(1)}$.

Os artigos selecionados apresentam a habilidade da liderança que se faz produtiva em estratégias práticas de aprendizagem experiencial, que são capazes de melhorar a liderança envolvendo a equipe interprofissional| ${ }^{(19,39,43,45)}$. Essas relações são importantes para o futuro profissional se inserir no campo de atuação, torna-o apto a assumir posições tendo em vista o bem-estar da comunidade, e implica na distribuição de poder por parte dos enfermeiros.

A aquisição de poder por enfermeiros e dos demais trabalhadores é fenômeno grupal na liderança, que envolve a influência social de um indivíduo sobre os demais. Nesse sentido, um estudo ${ }^{(10)}$ abordou a liderança na atividade de delegação e supervisão na prática do enfermeiro, evidenciando o trabalho em grupo, ressaltando e valorizando as competências individuais, as observações, o poder na equipe, fazendo com que cada membro reconheça o propósito e o significado de seu trabalho. É possível sinalizar para o enfermeiro as possibilidades de desempenhar esse novo papel de líder, orientado para o futuro, mais flexível, dinâmico ${ }^{(12,46-47)}$.

Alguns artigos examinados apontam que não há mais como negar a importância da função de liderar na atuação do enfermeiro, que, exercida sob orientação de novos padrões e valores, poderá trazer para as organizações de saúde, os serviços de enfermagem, a classe profissional e principalmente o cliente, benefícios que privilegiam assistência segura e com qualidade em interação humana constante ${ }^{(19,39,43,45)}$. A importância de uma relação com empatia foi tratada em um artigo que utilizou a aprendizagem experiencial, por meio da simulação com pacientes estomizados, para ensinar aos estudantes como realizar o cuidado de enfermagem em uma relação de empatia com seus pacientes ${ }^{(48)}$.

\section{Administração e gerenciamento}

De acordo com as DCN, no que tange à administração e ao gerenciamento, os profissionais devem ser capazes de tomar iniciativa, gerir recursos humanos e materiais, assim como liderar as equipes, dentre outras ${ }^{(1)}$. Por essa razão, a aprendizagem experiencial pode conduzir ao bom exercício da administração e do gerenciamento e se relacionar com outras competências e habilidades gerais como liderança, tomada de decisões e comunicação.

As atividades que envolvem a administração e o gerenciamento em Enfermagem estão presentes no processo de trabalho do enfermeiro e por isso o desenvolvimento de tais competências é essencial na formação desses profissionais. Para isso, os profissionais devem possuir um conjunto de conhecimentos relacionados à gestão de pessoas, planejamento, interação entre indivíduos e tomada de decisões ${ }^{(49)}$.

Nos artigos analisados, a competência da administração e o gerenciamento foram trabalhados na perspectiva do gerenciamento de recursos materiais e humanos, assim como no planejamento de ações em saúde. Ademais, as estratégias de aprendizagem experiencial utilizadas foram a simulação e a educação permanente e continuada ${ }^{(12,14,28,47)}$.

\section{Educação permanente}

A educação permanente versa sobre a capacidade dos indivíduos de aprender continuamente, tanto durante a sua formação, quanto no seu exercício profissional( ${ }^{(1)}$. Nos artigos analisados, a aprendizagem experiencial foi utilizada no treinamento de estudantes nos níveis de graduação e pós graduação, no aprendizado em serviço, assim como para 


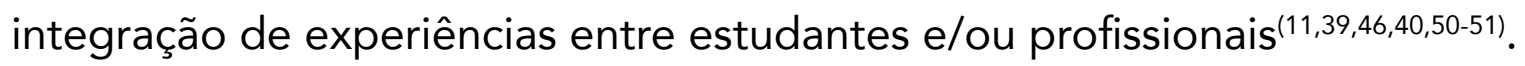

No âmbito do Sistema Único de Saúde (SUS), a Educação Permanente ressalta que os indivíduos devem ser ativos e aptos para serem capazes de aprender a aprender, aprender a ser, aprender a conviver e aprender a fazer ${ }^{(4)}$. Estes pilares da educação foram evidenciados por estudos envolvendo o aprender a aprender ${ }^{(12,18,51)}$ e o aprender a fazer $r^{(19,21,35,45-46,50,52-53)}$ e pelo Ciclo de Aprendizagem de Kolb(54-55).

Diante da complexidade dos pilares da educação e da diversidade apresentada nos estilos de aprendizagem de $\mathrm{Kolb}^{(5)}$, a aprendizagem em serviço pode ser estratégia para otimizar a formação em Enfermagem. Estudos com estudantes de graduação em Enfermagem, realizados na atenção básica, apontaram que o aprendizado em serviço pode contribuir para melhor compreensão dos determinantes sociais da saúde de uma comunidade, para identificação dos impactos das práticas de Enfermagem e para o desenvolvimento do senso de justiça social ${ }^{(19,40,46,50,53,56-57)}$.

Ressalta-se que os cenários do SUS são considerados espaços passíveis de integração entre ensino-serviço-comunidade, favorecendo a formação de recursos humanos, a educação e o desenvolvimento de pesquisas ${ }^{(4,8)}$. Ademais, as relações estabelecidas entre comunidade, estudantes, docentes e profissionais traz benefícios mútuos para todos os envolvidos em virtude dos vínculos construídos ${ }^{(54)}$, assim como a formação de profissionais mais qualificados para a atenção e produção de conhecimento em saúde.

\section{CONCLUSÃO}

A revisão integrativa possibilitou sistematizar o conhecimento produzido no nível nacional e internacional, com o objetivo de conhecer as contribuições da aprendizagem experiencial no ensino da Enfermagem, enfatizando a simulação como metodologia mais abordada nos artigos selecionados.

Foi possível evidenciar a aprendizagem experiencial como estratégia de ensino e aprendizagem na enfermagem e suas contribuições alcançam as competências e habilidades gerais definidas pelas DCN. A habilidade de Atenção à saúde foi a mais abordada nos estudos, promovendo o comprometimento e a responsabilidade de todos os agentes envolvidos na aprendizagem; a Tomada de Decisão foi atingida por meio de pensamento crítico e com base nos julgamentos clínicos, apontando que para aprender a pensar criticamente é necessário estimular o ato reflexivo.

Já a Comunicação compreendeu comunicação interprofissional e comunicação entre estudantes/profissionais e indivíduos/famílias; a Liderança se fez produtiva em estratégias práticas de aprendizagem experiencial que são capazes de melhorar a liderança envolvendo a equipe interprofissional; na Administração e Gerenciamento, a aprendizagem foi trabalhada para gerenciar recursos materiais e humanos, assim como para o profissional planejar de ações em saúde.

Destaca-se que aprendizagem experiencial defende a educação em constante transformação, influenciada pelo contexto social do indivíduo, que é capaz de criar e recriar o seu próprio conhecimento através da experiência. Neste sentido, a simulação possibilitou vivenciar experiências em diversos artigos, de forma segura e pedagogicamente planejada.

\section{REFERÊNCIAS}


2. Cunha M, Ribeiro O, Vieira $C$, Pinto $F$, Alves $L$, Santos $R$, et al. Atitudes do enfermeiro em contexto de ensino clínico: uma revisão da literatura. Millenium. [Internet]. 2010 [acesso em 02 set 2017]; 38(15). Disponível em: https://revistas.rcaap.pt/millenium/article/view/8262.

3. Ministério da Saúde (BR). Educação Permanente em Saúde: um movimento instituinte de novas práticas no Ministério da Saúde: Agenda 2014. [Internet]. Brasília: Ministério da Saúde, 2014 [acesso em 20 nov 2017]. Disponível em: http://bvsms.saude.gov.br/bvs/publicacoes/educacao permanente saude movimento instituinte.pdf.

4. Santos EO dos, Nunes CK, Demarco D de A, Amestoy SC. Aprendizagem baseada em problemas no ensino da enfermagem. Rev. Contexto Saúde [Internet]. 2017 [acesso em 02 set 2017]; 17(32). Disponível em: https://doi.org/10.21527/2176-7114.2017.32.55-66.

5. Kolb DA. Experiential learning: experience as the source of learning and development. Englewood Cliffs, NJ: Prentice Hall; 1984.

6. Pimentel A. A teoria da aprendizagem experiencial como alicerce de estudos sobre desenvolvimento profissional. Estud. psicol. [Internet]. 2007 [acesso em 02 set 2017]; 12(2). Disponível em: http://dx.doi. org/10.1590/S1413-294X2007000200008.

7. Ganong LH. Integrative review of nursing research. Res Nurs Health. [Internet]. 1987. [acesso em 02 set 2017]; 10(1). Disponível em: https://doi.org/10.1002/nur.4770100103.

8. Ministério da Saúde (BR). A proposta da CIT de mudança na Política Nacional de Atenção Básica PNAB. Nota técnica No 30/2017. [Internet]. Brasília, 24 de agosto de 2017. [acesso em 02 set 2017]. Disponível em: http://www.cnm.org.br/cms/biblioteca/NT 302017 Proposta CIT PNAB.pdf.

9. Lewis C, Reid J, McLernon Z, Ingham R, Traynor M. The impact of a simulated intervention on attitudes of undergraduate nursing and medical students towards end of life care provision. Bmc Palliative Care [Internet]. 2016 [acesso em 23 ago 2017]; 15. Disponível em: https://doi.org/10.1186/s12904-016-0143-2.

10. Wath Van der AE, Toit PH du. Learning end-of-life care within a constructivist model: Undergraduate nursing students' experiences. Curationis [Internet]. 2015 [acesso em 23 ago 2017]; 38(2). Disponível em: https://doi.org/10.4102/curationis.v38i2.1537.

11. Doolen J, Giddings M, Johnson M, Nathan GG de, Badia LO. An evaluation of mental health simulation with standardized patients. Int. J. Nurs. Educ. Scholarsh. [Internet]. 2014 [acesso em 23 ago 2017]; 11. Disponível em: https://doi.org/10.1515/ijnes-2013-0075.

12. Breen $H$, Jones $M$. Experiential learning: using virtual simulation in an online RN-to-BSN program. J contin educ nurs. [Internet]. 2015 [acesso em 23 ago 2017]; 46(1):27-33. Disponível em: https://doi. org/10.3928/00220124-20141120-02.

13. Shinnick MA, Woo MA. Learning style impact on knowledge gains in human patient simulation. Nurse educ today [Internet]. 2015 [acesso em 23 ago 2017]; 35(1):63-7. Disponível em: https://doi.org/10.1016/j. nedt.2014.05.013.

14. Ildarabadi E, Karimi Moonaghi $H$, Heydari A, Taghipour A, Abdollahimohammad A. Vaccination learning experiences of nursing students: a grounded theory study. J Educ Eval Health Prof. [Internet]. 2015 [acesso em 23 ago 2017]; 12. Disponível em: http://dx.doi.org/10.3352/jeehp.2015.12.29.

15. Grace S, Innes E, Patton N, Stockhausen L. Ethical experiential learning in medical, nursing and allied health education: A narrative review. Nurse educ today [Internet] 2017 [acesso em 23 ago 2017]; 51:2333. Disponível em: https://doi.org/10.1016/j.nedt.2016.12.024.

16. Ghazivakili Z, Norouzi Nia R, Panahi F, Karimi M, Gholsorkhi H, Ahmadi Z. The role of critical thinking skills and learning styles of university students in their academic performance. J adv med educ prof. [Internet]. 2014 [acesso em 23 ago 2017]; 2(3):95-102. Disponível em: https://www.ncbi.nlm.nih.gov/pmc/ articles/PMC4235550/pdf/jamp-2-95.pdf. 
17. Weideman $Y L$, Culleiton AL. A virtual pregnancy for pre-licensure nursing students: Nine months up and close. Nurs. educ. perspect. [Internet]. 2014 [acesso em 23 ago 2017]; 35(6):410-4. Disponível em: http://dx.doi.org/10.5480/11-601.1.

18. Oliveira SN de, Prado ML do, Kempfer SS, Waterkemper R, Morera JAC, Bernardi MC. A pedagogia por trás da experiência clínica simulada: uma percepção de estudantes de enfermagem. Rev iberoam educ invest Enferm [Internet]. 2015 [acesso em 23 ago 2017]; 5(3). Disponível em: http://www. enfermeria21.com/revistas/aladefe/articulo/173/.

19. King AEA, Conrad M, Ahmed RA. Improving collaboration among medical, nursing and respiratory therapy students through interprofessional simulation. J interprof care. [Internet]. 2013 [acesso em 23 ago 2017]; 27(3):269-71. Disponível em: https://doi.org/10.3109/13561820.2012.730076.

20. Smith Z, Leslie G, Wynaden D. Experiential learning not enough for organ procurement surgery: implications for perioperative nursing education. Prog Transplant. [Internet]. 2015 [acesso em 23 ago 2017]; 25(4):339-50. Disponível em: https://doi.org/10.7182/PIT2015689.

21. Jamison T, Lis GA. Engaging the learner by bridging the gap between theory and clinical competence: The impact of concept mapping and simulation as innovative strategies for nurse-sensitive outcome indicators. Crit. care nurs. clin. North Am. [Internet]. 2014 [acesso em 23 ago 2017]; 49(1):69. Disponível em: https://doi.org/10.1016/j.cnur.2013.11.004.

22. Lau Y, Wang W. Development and evaluation of a learner-centered educational summer camp program on soft skills for baccalaureate nursing students. Nurse Educ. [Internet]. 2014 [acesso em 23 ago 2017]; 39(5):246-51. Disponível em: https://www.ncbi.nlm.nih.gov/pubmed/24978015.

23. Fogg L, Carlson-Sabelli L, Carlson K, Giddens J. The perceived benefits of a virtual community effects of learning style, race, ethnicity, and frequency of use on nursing students. Nurs Educ Perspect [Internet]. 2013 [acesso em 23 ago 2017]; 34(6):390-4. Disponível em: https://www.ncbi.nlm.nih.gov/ pubmed/24475600.

24. Hutchinson TL, Janiszewski Goodin H. Nursing student anxiety as a context for teaching/learning. J Holist Nurs [Internet]. 2013 [acesso em 23 ago 2017]; 31(1):19-24. Disponível em: https://doi. org/10.1177/0898010112462067.

25. Piña-Jiménez I, Amador-Aguilar R. La enseñanza de la enfermería con simuladores, consideraciones teórico-pedagógicas para perfilar un modelo didáctico. Enferm. univ. [Internet]. 2015 [acesso em 23 ago 2017]; 12(3):152-9. Disponível em: http://dx.doi.org/10.1016/j.reu.2015.04.007.

26. Brehmer LC de F, Ramos FRS. The healthcare model in training for nursing: experiences and perceptions. Interface (Botucatu). [Internet]. 2016 [acesso em 30 ago 2017]; 20(56). Disponível em: http:// dx.doi.org/10.1590/1807-57622015.0218.

27. Celik Y, Ceylantekin Y, Kilic I. The evaluation of simulation market in nursing education and the determination of learning style of students. Int. J. of Health Sci. [Internet]. 2017 [acesso em 23 ago 2017]; 11(1):13-8. Disponível em: https://www.ncbi.nlm.nih.gov/pmc/articles/PMC5327665/.

28. Arveklev SH, Wigert $H$, Berg $L$, Burton $B$, Lepp $M$. The use and application of drama in nursing education-an integrative review of the literature. Nurse educ today. [Internet]. 2015 [acesso em 23 ago 2017]; 35(7):e12-7. Disponível em: https://doi.org/10.1016/j.nedt.2015.02.025.

29. Chiang VC, Chan SS. An evaluation of advanced simulation in nursing: a mixed-method study. Collegian [Internet]. 2014 [acesso em 23 ago 2017]; 21(4):257-65. Disponível em: https://doi. org/10.1016/j.colegn.2013.05.003.

30. Andersen HE, Larsen KV. Sculpting with people: An experiential learning technique. Nurse Educ Pract. [Internet]. 2015 [acesso em 23 ago 2017]; 15(6):556-60. Disponível em: https://doi.org/10.1016/j. nepr.2015.07.014.

31. Chmil JV, Turk M, Adamson K, Larew C. Effects of an experiential learning simulation design on clinical nursing judgment development. Nurse Educ. [Internet]. 2015 [acesso em 23 ago 2017]; 40(5):228-32. Disponível em: https://doi.org/10.1097/NNE.0000000000000159. 
32. Oliveira SN de, Prado ML do, Kempfer SS, Martini JG, Caravaca-Morera JA, Bernardi MC. Experiential learning in nursing consultation education via clinical simulation with actors: Action research. Nurse educ. today. [Internet]. 2015 [acesso em 23 ago 2017]; 35(2):e50-e4. Disponível em: https://doi.org/10.1016/j. nedt.2014.12.016.

33. Lavoie P, Pepin J, Cossette S. Development of a post-simulation debriefing intervention to prepare nurses and nursing students to care for deteriorating patients. Nurse educ pract. [Internet]. 2015 [acesso em 23 ago 2017]; 15(3):181-91. Disponível em: http://dx.doi.org/10.1016/j.nepr.2015.01.006.

34. McNamara N. Preparing students for clinical placements: The students perspective. Nurse educ pract. [Internet]. 2015 [acesso em 23 ago 2017]; 15(3):196-202. Disponível em: https://doi.org/10.1016/j. nepr.2014.11.011.

35. Zigmont JJ, Wade A, Edwards T, Hayes K, Mitchell J, Oocumma N. Utilization of experiential learning, and the learning outcomes model reduces RN orientation time by more than $35 \%$. Clin Simul Nurs [Internet]. 2015 [acesso em 23 ago 2017]; 11(2):79-94. Disponível em: https://doi.org/10.1016/j. ecns.2014.11.001.

36. Sordi MRL de, Bagnato MHS. Subsídios para uma formação profissional crítico-reflexiva na área de saúde: o desafio da virada do século. Rev. Latino-am. Enferm. [Internet]. 1998 [acesso em 30 set 2017]; 6(2). Disponível em: http://dx.doi.org/10.1590/S0104-11691998000200012.

37. Koivisto J-M, Niemi H, Multisilta J, Eriksson E. Nursing students' experiential learning processes using an online 3d simulation game. Educ. inf. technol. [Internet]. 2017 [acesso em 23 ago 2017]; 22(1):383-98. Disponível em: https://doi.org/10.1007/s10639-015-9453-x.

38. Ministério da Saúde (BR). Síntese de Evidências para Políticas de Saúde: estimulando o uso de evidências científicas na tomada de decisão [Internet]. Brasília: Ministério da Saúde; 2015 [acesso em 20 nov 2017]. Disponível em: http://bvsms.saude.gov.br/bvs/publicacoes/sintese evidencias politicas saude 1ed.pdf.

39. Doolen J, Giddings M, Johnson M, Guizado de Nathan G, O Badia L. An evaluation of mental health simulation with standardized patients. Int. j. nurs. educ. scholarsh.. [Internet]. 2014 [acesso em 23 ago 2017]; 11. Disponível em: https://doi.org/10.1515/ijnes-2013-0075.

40. Townsend L, Gray J, Forber J. New ways of seeing: Nursing students experiences of a pilot service learning program in Australia. Nurse educ pract. [Internet]. 2016 [acesso em 23 ago 2017]; 16(1):60-5. Disponível em: https://doi.org/10.1016/j.nepr.2015.08.004.

41. Ministério da Saúde (BR). Humaniza SUS: documento base para gestores e trabalhadores do SUS. [Internet] 4. ed. Brasília: Ministério da Saúde; 2010 [acesso em 20 nov 2017]. Disponível em: http://bvsms. saude.gov.br/bvs/publicacoes/humanizasus documento gestores trabalhadores sus.pdf.

42. Henoch I, Ung EJ, Ozanne A, Falk H, Falk K, Sarenmalm EK, et al. Nursing students' experiences of involvement in clinical research: an exploratory study. Nurse Educ Pract. [Internet]. 2014 [acesso em 23 ago 2017]; 14(2):188-94. Disponível em: https://doi.org/10.1016/j.nepr.2013.07.009.

43. Poore JA, Cullen DL, Schaar GL. Simulation-based interprofessional education guided by Kolb's experiential learning theory. Clin Simul Nurs. [Internet]. 2014 [acesso em 23 ago 2017]; 10(5):E241-E7. Disponível em: https://doi.org/10.1016/j.ecns.2014.01.004.

44. Almeida É de, Piexak DR, Ilha S, Marchior MRCT, Backes DS. Liderança do enfermeiro responsável técnico: um fazer necessário para o exercício profissional. Rev. pesqui. cuid. fundam. (Online). [Internet]. 2014 [acesso em 23 ago 2017]; 6 (3). Disponível em: https://doi.org/10.9789/2175-5361.2014v6n3p998.

45. Onge J St, Hodges T, McBride M, Parnell R. An innovative tool for experiential learning of nursing quality and safety competencies. Nurse Educ. [Internet]. 2013 [acesso em 23 ago 2017]; 38(2):71-5. Disponível em: https://www.ncbi.nlm.nih.gov/pubmed/23407197.

46. Crowder SJ, Ironside PM, Cangany M, Roddy WS. Implementation of a state legislative fellowship for doctor of nursing practice students. Nurse Educ. [Internet]. 2016 [acesso em 23 ago 2017]; 41(3):156-9. 
Disponível em: https://www.ncbi.nlm.nih.gov/pubmed/26771941.

47. Jordan SD, Collins-Yoder AS. Mock board hearing: giving students new insights through experiential learning. Clin Simul Nurs [Internet]. 2014 [acesso em 23 ago 2017]; 10(12):630-3. Disponível em: http:// dx.doi.org/10.1016/j.ecns.2014.09.005.

48. Maruca AT, Díaz DA, Kuhnly JE, Jeffries PR. Enhancing empathy in undergraduate nursing students: An experiential ostomate simulation. Nurs Educ Perspect [Internet]. 2015 [acesso em 23 ago 2017]; 36(6):367-71. Disponível em: https://www.ncbi.nlm.nih.gov/pubmed/26753295.

49. Benito GAV, Tristão KM, Paula ACFS de, Santos MA dos, Ataide, LJ, Lima R de CD, et al. Desenvolvimento de competências gerais durante o estágio supervisionado. Rev. bras. enferm. [Internet]. 2012 [acesso em 30 set 2017]; 65(1). Disponível em: http://dx.doi.org/10.1590/S003471672012000100025.

50. Schmidt NA, Brown JM. Service learning in undergraduate nursing education: strategies to facilitate meaningful reflection. J Prof Nurs. [Internet]. 2016 [acesso em 23 ago 2017]; 32(2):100-6. Disponível em: https://doi.org/10.1016/j.profnurs.2015.06.006.

51. Rassin M, Kurzweil Y, Maoz Y. Identification of the learning styles and "On-the-Job" learning methods implemented by nurses for promoting their professional knowledge and skills. Int J Nurs Educ Scholarsh [Internet]. 2015 [acesso em 23 ago 2017]; 12(1). Disponível em: https://doi.org/10.1515/ijnes-2015-0006.

52. Day L, Turner K, Anderson RA, Mueller C, McConnell ES, Corazzini KN. Teaching delegation to RN students. J Nurs Regul [Internet]. 2014 [acesso em 23 ago 2017]; 5(2):10-5. Disponível em: https://doi. org/10.1016/S2155-8256(15)30083-1.

53. Salmon S, Wang XB, Seetoh T, Lee SY, Fisher DA. A novel approach to improve hand hygiene compliance of student nurses. Antimicrob Resist Infect Control [Internet]. 2013 [acesso em 23 ago 2017]; 2. Disponível em: https://doi.org/10.1186/2047-2994-2-16.

54. Mitchell EKL, James S, D'Amore A. How learning styles and preferences of firstyear nursing and midwifery students change. Aust J Educ [Internet]. 2015 [acesso em 23 ago 2017]; 59(2):158-68.

Disponível em: https://doi.org/10.1177/0004944115587917.

55. El-Gilany A-H, Abusaad FES. Self-directed learning readiness and learning styles among Saudi undergraduate nursing students. Nurse educ today [Internet]. 2013 [acesso em 23 ago 2017]; 33(9):10404. Disponível em: https://doi.org/10.1016/j.nedt.2012.05.003.

56. McGuire M, Goldstein C, Claywell L, Patton R. Analysis of student reflections of experiential learning in nursing health policy courses. Nurse Educ. [Internet]. 2017 [acesso em 23 ago 2017]; 42(2):95-9.

Disponível em: https://www.ncbi.nlm.nih.gov/pubmed/27580301.

57. Main EE, Garrett-Wright D, Kerby M. Nursing student voices: reflections on an international service learning experience. Ky Nurse. [Internet]. 2013 [acesso em 23 ago 2017]; 61(1):10-1. Disponível em: https://www.ncbi.nlm.nih.gov/pubmed/23367657.

Recebido: 19/04/2018

Finalizado: 30/10/2019

Autor Correspondente:

Paula Bresolin

Universidade Federal de Santa Catarina

R. José Dutra, 70 - 88036-205 - Florianópolis, SC, Brasil

E-mail: paulabresolin5@gmail.com 
Contribuição dos autores:

Contribuições substanciais para a concepção ou desenho do estudo; ou a aquisição, análise ou interpretação de dados do estudo - JGM, ISG, MHPAB

Elaboração e revisão crítica do conteúdo intelectual do estudo - JR

Aprovação da versão final do estudo a ser publicado - DDL

Responsável por todos os aspectos do estudo, assegurando as questões de precisão ou integridade de qualquer parte do estudo - PB 